...lower thyroid

function is

associated

with an

increased risk

of developing

NAFLD...

\title{
Poor thyroid function linked to NAFLD
}

Hypothyroidism has been implicated in the development of nonalcoholic fatty liver disease (NAFLD), but these data have given conflicting results. Now, in data from a new study, poor thyroid function is shown to be significantly associated with an increased risk of developing NAFLD.

"Prior studies regarding the association between thyroid function and NAFLD risk have yielded controversial results, mainly due to small sample sizes and cross-sectional designs," explains study author Arjola Bano.

The team used data from The Rotterdam Study for what is the first prospective investigation assessing thyroid function and the risk of NAFLD. In total, 9,419 individuals with baseline thyroid function and fatty liver index measurements were included; the presence of NAFLD was assessed by ultrasonography at follow-up.
At $\sim 10$ years follow-up, increased levels of free $\mathrm{T}_{4}$ were associated with a decreased risk of NAFLD (OR 0.33, $95 \%$ CI $0.22-0.48$ per $1 \mathrm{ng} / \mathrm{dl}$ of free $\mathrm{T}_{4}$ ). Those in the lowest free $\mathrm{T}_{4}$ tertile had 1.31-times greater risk of developing NAFLD than those in the highest tertile (95\% CI 1.11-1.56).

These risks were independent of possible confounding factors, such as cardiovascular risk factors.

Conversely, those individuals with increased TSH levels were at greater risk of developing NAFLD (OR 1.09, 95\% CI 1.01-1.19 per 1 $\log \mathrm{TSH}$ ), although this risk was slightly attenuated when adjusted for cardiovascular risk factors (OR 1.07). Importantly, the team found a significant decreasing trend in NAFLD risk across patients from clinical hypothyroidism to clinical hyperthyroidism $(P=0.003)$.

Finally, Bano and colleagues also assessed the likelihood of developing NAFLD with liver stiffness, which might indicate the development of fibrosis. Overall, the risk of developing NAFLD with increased liver stiffness was 2.30-fold higher in patients with subclinical hypothyroidism than in those with euthyroidism, which remained significant after additional adjustments for cardiovascular risk factors were included in the analysis.

"Our large prospective populationbased study ... demonstrates that lower thyroid function is associated with an increased risk of developing NAFLD," says Bano. She concludes, "our findings highlight the need for future investigations on possible preventive or therapeutic interventions, such as screening of thyroid function in patients with NAFLD or treatment decisions in subclinical thyroid dysfunction."

Tim Geach

ORIGINAL ARTICLE Bano, A. et al. Thyroid function and the risk of non-alcoholic fatty liver disease: The Rotterdam Study.J.Clin. Endocrinol. Metab. http:// dx.doi.org/10.1210/jc.2016-1300 (2016) 